\title{
Optimization of the non-axisymmetric stator casing of a 1.5 stage axial turbine
}

\author{
Hakim Kadhim $^{\text {a,b }}$, Aldo Rona ${ }^{\text {a,*, J. Paul Gostelow }}{ }^{\text {a }}$ \\ Department of Engineering, University of Leicester, LE1 7RH, United Kingdom \\ Katrin Leschke ${ }^{c}$ \\ ${ }^{\mathrm{b}}$ Al-Furat Al-Awsat Technical University, Technical Institute, Al-Dewaniyah, Iraq \\ ${ }^{\mathrm{c}}$ Department of Mathematics, University of Leicester, LE1 7RH, United Kingdom \\ *Corresponding author: phone: +44 116252 2510; fax: +44 116252 2619; e-mail: ar45@leicester.ac.uk.
}

\begin{abstract}
The interaction of secondary flows with the main passage flow in turbomachines results in entropy generation and in aerodynamic loss. This loss source is most relevant to low aspect ratio blades. One approach for reducing this flow energy loss is by end-wall contouring. However, limited work has been reported on using non-axisymmetric end-walls at the stator casing and on its interaction with the tip leakage flow. In this paper, a non-axisymmetric end-wall design method for the stator casing is implemented through a novel surface definition, towards mitigating secondary flow losses. This design is tested on a three-dimensional axial turbine RANS model built in OpenFOAM Extend 3.2, with $k-\omega$ SST turbulence closure. Flow analysis confirm the foundations of the new surface definition approach, which is implemented using Alstom Process and Optimization Workbench (APOW) software. Computer-based optimization of the surface topology is demonstrated towards automating the design process of axial turbines in an industrial design workflow. The design is optimized using the total pressure loss across the first stator and across the full stage, as the target function. Numerical predictions of the 1.5 stage axial turbine show the positive impact of the optimized casing design on the efficiency that increases by $0.69 \%$ against the benchmark axisymmetric stage from RTWH Aachen, which is validated against experiment. The new non-axisymmetric casing is also beneficial at off-design condition. The effective mitigation of the secondary flows is predicted to give a $0.73 \%$ efficiency gain off-design.
\end{abstract}


Keywords: Axial turbine; non-axisymmetric end wall design; secondary flows; aerodynamic loss. 


\section{NOMENCLATURE}

$\begin{array}{ll}f & \text { Beta probability density function (-) } \\ R_{d} & \text { Groove radial depth (mm) } \\ R & \text { Specific gas constant }\left(\mathrm{J} \mathrm{kg}^{-1} \mathrm{~K}^{-1}\right) \\ w & \text { Casing groove width (radians) } \\ g & \text { Polynomial function of degree } 4(-) \\ r & \text { Radial distance }(\mathrm{mm}) \\ r_{h} & \text { Hub radius (mm) } \\ r_{t} & \text { Casing radius (mm) } \\ r_{h t} & \text { Casing groove radius (mm) } \\ s & \text { Parametrized surface }(-) \\ x & \text { Axial coordinate (mm) } \\ \theta & \text { Pitchwise angular coordinate (radians) } \\ \theta_{g} & \text { Groove pitchwise angle (radians) } \\ \dot{\theta} & \text { Design shaft angular speed (r.p.m.) } \\ P_{i} & \text { Casing surface point (-) } \\ x_{i} & x \text {-coordinate of the } i^{t h} \text { point (mm) } \\ y_{i} & y \text {-coordinate of the } i^{t h} \text { point (mm) } \\ z_{i} & z \text {-coordinate of the } i^{\text {th }} \text { point (mm) } \\ \theta_{i} & \theta \text { coordinate of the } i^{\text {th }} \text { point (radians) } \\ h & \text { Blade span (mm) } \\ k & \left.\text { Specific turbulent kinetic energy (m }{ }^{2} \mathrm{~s}^{-2}\right) \\ y^{+} & \text {Dimensionless wall distance }(-)\end{array}$

\section{Greek symbols}

$\alpha$

$\alpha_{2}$

$\beta$

$\Gamma$

$\eta$

$\omega$

$\mu$

$\xi$

\section{Acronyms}

APOW

LE

NURBS

PS

SS

TE

UNFCCC
Shape factor (-)

Rotor exit absolute yaw angle (degrees)

Scale factor (-)

Gamma probability density function (-)

Minimum distance (mm)

Specific dissipation rate $\left(\mathrm{m}^{2} \mathrm{~s}^{-3}\right)$

Maximum groove depth location (-)

Groove path curve (-)

Alstom Process and Optimization Workbench

Leading edge

Non-Uniform Rational B-Spline

Pressure Side

Suction Side

Trailing Edge

United Nations Framework Convention on Climate Change 


\section{Introduction}

Mitigating techniques for secondary flows are commonly applied in the design of axial turbomachines to manage the performance loss due to them. These flows are responsible for approximately one third of the estimated total aerodynamic losses of an axial turbine [1]. Understanding the physics and ability to predict these secondary flow structures is the first step to control and reduce the loss and further achieve an increase in turbine efficiency. Such gain is critical for limiting the $\mathrm{CO} 2$ emissions towards meeting the UNFCCC emissions goals. The axial turbine is a key component in an industrial turbo-engine. The overall cycle efficiency is primarily affected by the turbine performance [2] and its gains have tangible societal, economic, and environmental impact.

A comprehensive and detailed description of secondary flows is presented in references [3-6]. Various methods have been developed to reduce secondary flow losses. For this purpose, end wall modifications are used and assessed by RANS simulations and experiments. Dossena et al. [7] tested numerically and experimentally a radially profiled axisymmetric casing for a turbine nozzle guide vane, showing a significant performance improvement with respect to a non-profiled casing. Moser et al. [8] designed an optimized radially profiled axisymmetric casing for a guide vane of a steam turbine stage that reduced the stage loss over a wide range of pressure ratios. Kumar and Govardhan [9] applied a streamwise end wall fence to reduce secondary flow losses in a linear turbine cascade. They predicted reductions in the exit flow angle deviation, secondary flow losses, and the magnitude and spanwise penetration of the passage vortex.

Other researchers pursued a non-axisymmetric end wall design approach for reducing secondary flow losses. A numerical simulation of a nozzle guide vane passage with a profiled hub was first performed by Rose [10]. This changed the passage cross-flow, reducing the loss. Hartland et al. 
[11] and Ingram et al. [12] tested a non-axisymmetric end wall design on a linear cascade and obtained about a $24 \%$ reduction in secondary flow loss. Brennan et al. [13], Harvey et al. [14] used non-axisymmetric contouring to obtain a one-third reduction in the end wall loss and a $0.59 \%$ increase in the stage efficiency in a high pressure turbine and a $0.9 \%$ efficiency increase in the low pressure turbine of the Rolls-Royce Trent 500 engine. Germain et al. [15] improved the efficiency of a one-and-half stage highly loaded axial flow turbine using non-axisymmetric end wall contouring that reduced the secondary losses and also weakened the mid-span flow losses. Schuepbach et al. [16] confirmed these efficiency improvements by a time-resolved experimental and numerical investigation of the flow physics, which highlighted the presence of reduced blade trailing edge shed vorticity in the contoured wall passage.

How the end wall geometry is parametrized has a crucial importance in the design process. Harvey et al. [17] adopted beta-spline and Fourier series based curves in the axial and circumferential directions to define the profiled end wall shapes. They demonstrated a reduction in secondary flows, in the secondary kinetic energy, and in the blade row exit angle deviations. Germain et al. [15] used a combination of a pitch-wise shape function and a stream-wise decay function that, when multiplied and scaled, define the contoured end wall surfaces. Praisner et al. [18] argued that there were disadvantages in using shape functions to parametrize a contoured end wall. Simple shape functions, such as a sinusoidal curve, imply a preconceived notion of the resulting geometry. Praisner et al. [18] therefore proceeded to parametrize their geometry using two-dimensional cubic splines in the pitchwise and streamwise directions. This removed some of the restrictions from the prescribed shape of the guide curves used in the previous work.

Many researchers have used computer-based optimization methods to enhance the system performance of axial and radial turbines [19-23]. This includes the application of computer-based 
optimization to contoured end-walls of axial turbines. For instance, Sun et al. [24] used an optimization technique based on combining end wall profiling parametrization, global optimization, and aerodynamic performance evaluation methods, lowering secondary flow and profile losses. Tang et al. [25] optimized the end wall of a one-and-half stage high-work axial turbine using a multi-island genetic algorithm, achieving $10.7 \%$ total pressure loss decrement across the first stator and an overall $0.4 \%$ stage efficiency increase.

The literature indicates important gaps in the knowledge of end-wall designs for axial turbines. There is currently no widespread consensus around a specific end wall treatment being best for enhancing the performance of axial turbines. Additionally, in the optimization process of a turbine, the number of the design variables is still large. This makes the optimization process computationally expensive. This work makes progress towards addressing these important gaps by introducing a new design of the turbine casing by a novel surface definition method. The new design uses a comparatively small number of free parameters that are shown by numerical modelling to give attractive increases in the stage isentropic efficiency.

In this paper, a baseline three-dimensional steady RANS k- $\omega$ SST model, with axisymmetric walls, is validated against experimental measurements from the Institute of Jet Propulsion and Turbomachinery at the Rheinisch-Westfälische Technische Hochschule (RWTH) Aachen, Germany, using the OpenFOAM 3.2-extend solver. ICEM CFD was used as the geometry and mesh generator.

A non-axisymmetric casing is then introduced, based on a novel surface definition method that draws from observations of the typical secondary flow pattern over the casing. The incoming boundary layer typically separates, creating a horseshoe vortex at the blade leading edge. The pressure side arm moves across towards the suction side, merging with the suction side and 
creating the passage vortex. By delaying the onset of this interaction, there is scope for reducing the passage vortex size and hence its associated loss. To achieve this, a parametric groove designed by a Beta distribution is used to guide the horseshoe vortex pressure side arm. This is the first application of the Beta function to defining the casing geometry of a turbomachine. The newly design is applied to a 1.5 stage axial turbine to examine its effect on the secondary flow features. The design technique is implemented using Alstom Process and Optimization Workbench (APOW) software, which makes it compatible with the design process at GE. The workflow is executed in batch mode and automated using APOW.

An analysis of the predicted flow is presented to verify the effectiveness of the groove in mitigating the interaction of secondary flow structures and their effect on the stage efficiency.

\section{Test Case}

Walraevens and Gallus [26] provide measurements in a 1.5 stage axial turbine that are used for establishing a baseline CFD model of the passage flow. This set-up was selected for evaluating the performance of the casing treatment as a 1.5 stage axial turbine represents the smallest sub-set of a multi-row turbine that is able to display the effects of the treatment on the stage where this is applied, as well as the effects on the flow downstream of it, through the second stator row, the flow through which is indicative of the flow quality through subsequent blade rows.

Measurements through the 1.5 stage turbine were acquired downstream of the exit plane of the first stator, the rotor, and the second stator. Both stators and the rotor are untwisted blades. The geometry of the second stator, the stagger angle, and the number of blades are the same as the first stator. The rotor blades are unshrouded with a tip clearance of $0.4 \mathrm{~mm}$. The low aspect ratio blading and the constant radius end walls design results in strong secondary flows. The second stator is 
clocked three degrees in the direction of rotation. The 1.5 stage is tested at a mass flow rate of 7 kg/s. Cascade and meridional plane schematics of the Aachen turbine are given in Fig. 1 and key design data are listed in Table 1.
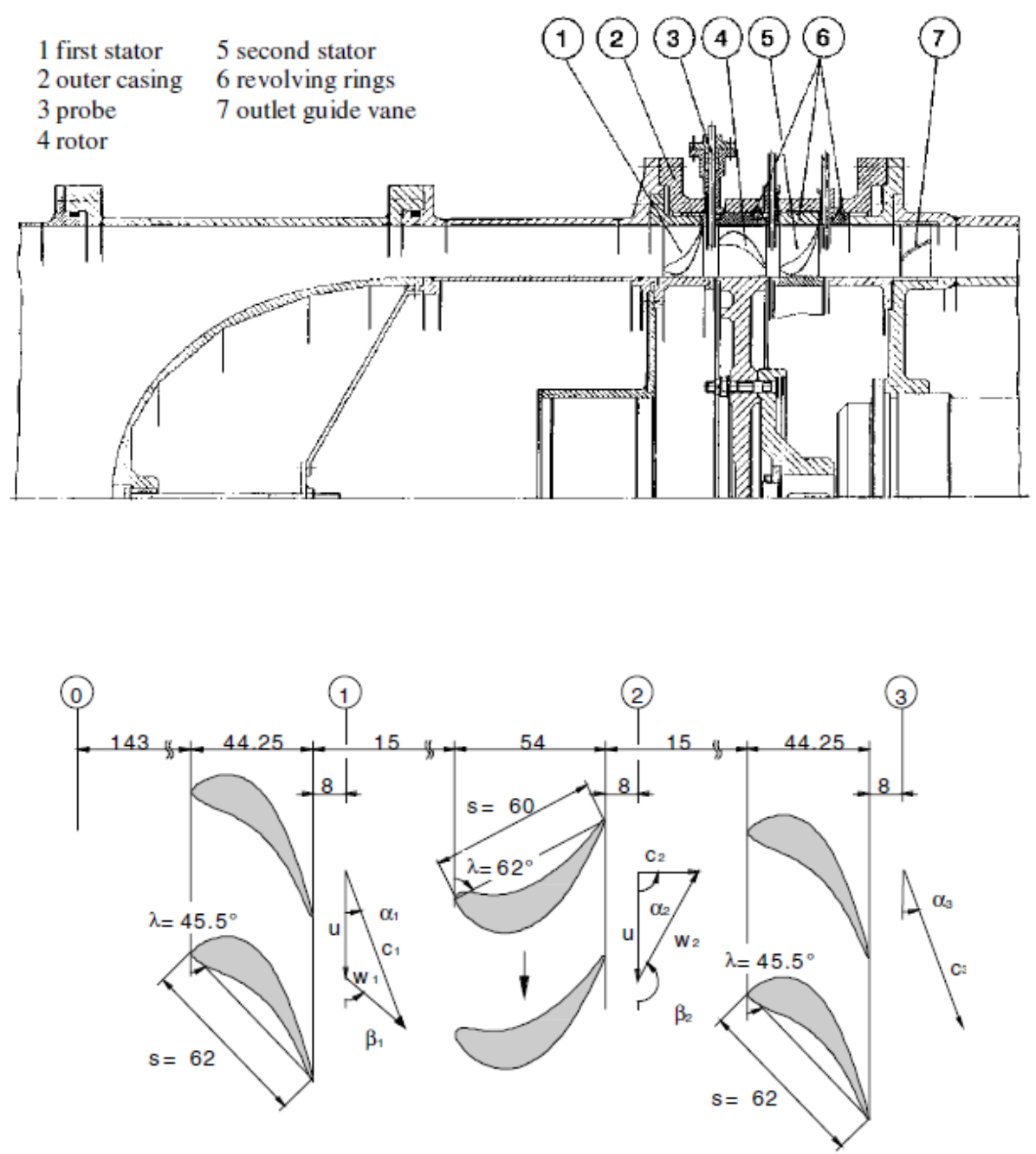

Fig. 1. Schematic of cross sectional view of the Aachen Turbine and detailed geometry [25]. 


\section{Table 1}

Design data of the turbine test case.

\begin{tabular}{l|c|c}
\hline \multirow{2}{*}{ Parameters } & \multicolumn{2}{|c}{ Values } \\
\cline { 2 - 3 } & $\begin{array}{c}\text { First and second } \\
\text { stator }\end{array}$ & Rotor \\
\hline Tip diameter & $600 \mathrm{~mm}$ & $600 \mathrm{~mm}$ \\
Hub diameter & $490 \mathrm{~mm}$ & $490 \mathrm{~mm}$ \\
Passage height, $h$ & $55 \mathrm{~mm}$ & $55 \mathrm{~mm}$ \\
Aspect ratio, $h / s$ & 0.887 & 0.917 \\
Blade number & 36 & 41 \\
Tip clearance & - & $0.4 \mathrm{~mm}$ \\
Midspan blade pitch, $t$ & $47.6 \mathrm{~mm}$ & $41.8 \mathrm{~mm}$ \\
Stator 1 and 2 absolute outflow angle $\alpha$ & $20.0^{\circ}$ & $90.0^{\circ}$ \\
Rotor relative inflow angle $\beta_{1}$ & - & $49.3^{\circ}$ \\
Rotor relative outflow angle $\beta_{2}$ & - & $151.2^{\circ}$ \\
Trailing edge thickness & $1.21 \mathrm{~mm}$ & $0.76 \mathrm{~mm}$ \\
\hline \hline
\end{tabular}

\section{CFD Method and Model Validation}

The Aachen turbine flow is investigated numerically by building a baseline three-dimensional steady RANS k- $\omega$ SST model. OpenFOAM 3.2 extend with the steadyCompressibleMRFFoam solver generates the flow solutions using mixing planes at the stator-rotor interface. The $k-\omega$ SST turbulence model was selected as it is computationally cheaper than a full Reynolds stress model for providing turbulence closure to the RANS equations. The model has been used extensively for RANS simulations of turbomachinery flows including flows exhibiting boundary layer separation [27]. It attempts to combine two of the most commonly used two-equation turbulence models: the $k-\omega$ model and the $k-\varepsilon$ model. The general idea is for the $k-\omega$ SST model to follow the behaviour of the $k-\omega$ model in the near-wall regions and that of the $k-\varepsilon$ model away from the walls. By using $k-\omega$ SST model, boundary layers through the passage can be predicted using the good turbulent boundary layer modelling behaviour of the $k-\omega$ model, in which the trend of $\omega$ at the wall is numerically more stable than that of $\varepsilon$. The prediction of the 
blade wakes takes advantage of the better behaviour of the $k-\varepsilon$ model in free mixing layers.

The computational domain is pitchwise periodic and one blade pitch around the annulus is modelled. The stator blade profile is stacked radially at the trailing edge, while the rotor is stacked radially at its centroid that is located at $x=25.265 \mathrm{~mm}$ and $y=13.456 \mathrm{~mm}$. The computational domain was generated using ANSYS ICEM CFD. The blading profiles are tabulated in Walraevens and Gallus [26] as 116 points for the stator and 133 points for the rotor, in two dimensions. These are mapped to a cylindrical reference system $(x, r, \theta)$, where $x$ is the axis of rotation, $r$ the radial distance from $x$, and $\theta=0$ is through the stator blade trailing edge.

Each passage volume is initially divided into three hexahedral sub-domains, as shown in the Fig. 2 (a). ANSYS ICEM CFD 16.2 is used to discretize the computational domain using hexahedral unit volumes and to export the mesh as unstructured to ANSYS Fluent. The mesh is converted to OpenFOAM format for use by the steadyCompressibleMRFFoam solver.

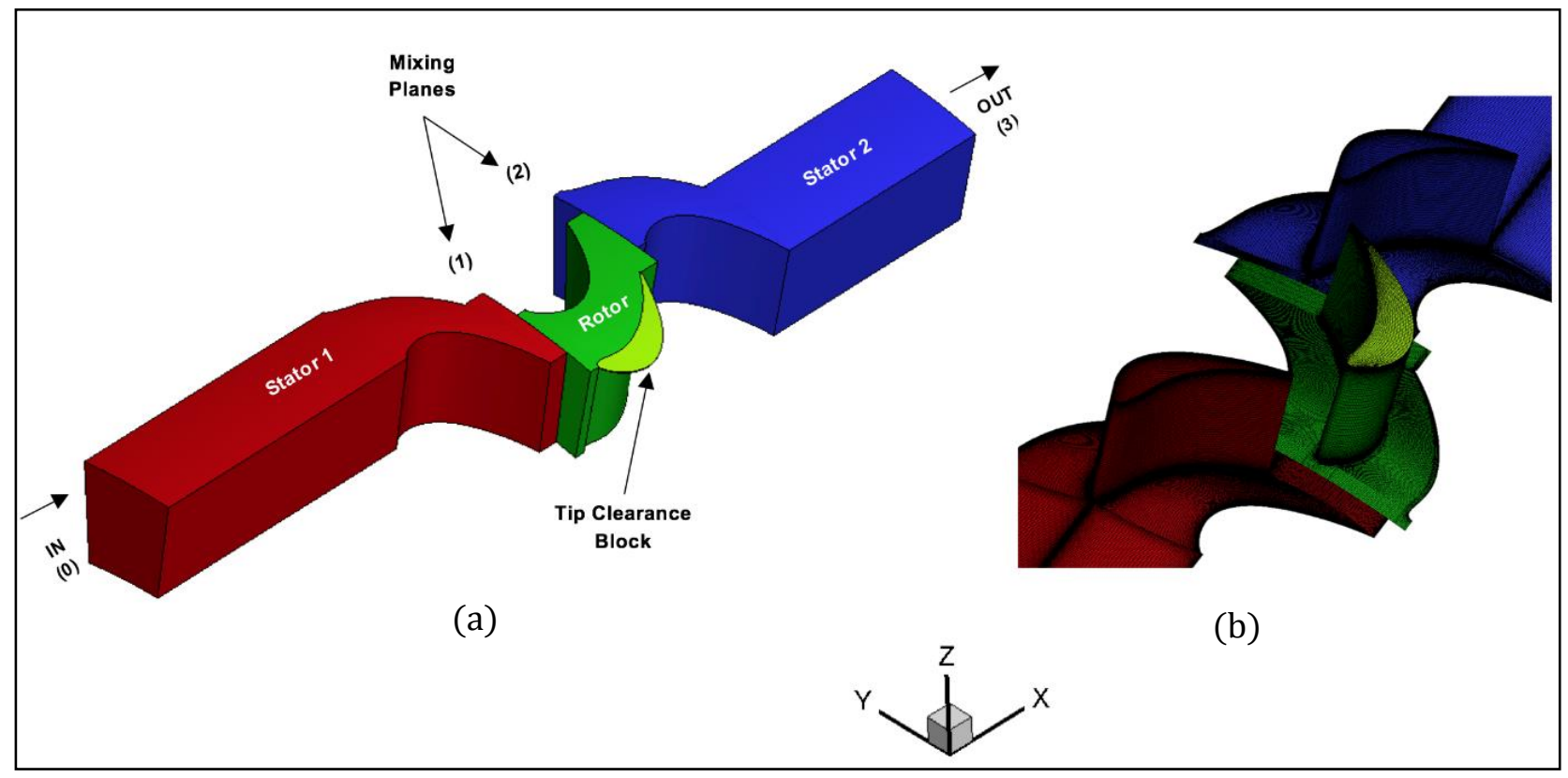

Fig. 2. (a) Schematic of the 1.5 stage turbine flow passage, (b) Detail of the 3-D computational mesh. 
A simple H-mesh topology is applied to the first and second stators blades passages and O-mesh topology is applied to the rotor blade passage. The rotor tip clearance is accounted for by adding an extra O-type block as shown in Fig. 2 (a). The mesh points are clustered close to the solid walls to resolve the boundary layer flow over the blade, the hub, and the casing. Fig. 2 (b) shows the resulting computational mesh. This covers one blade passage per row and a second passage is added in Fig. 2 (b) for clarity. Fig. 3 shows the average value of $\mathrm{y}^{+}$obtained by this clustering over the casing, from a number of CFD solutions with different non-axisymmetric casing parameter values. These CFD solutions, shown by the filled circles, are used to optimize the casing geometry as described in section 5. The average value of $y^{+}$of the optimized casing is shown by the filled diamond. By clustering the mesh points close to the wall, Fig. 3 shows that a near-wall resolution of $\mathrm{y}^{+} \approx 1$ is achieved and maintained through the optimization process. This is the same $\mathrm{y}^{+}$magnitude as that used by Menter et al. [28] for modelling a turbine stator guide vane.

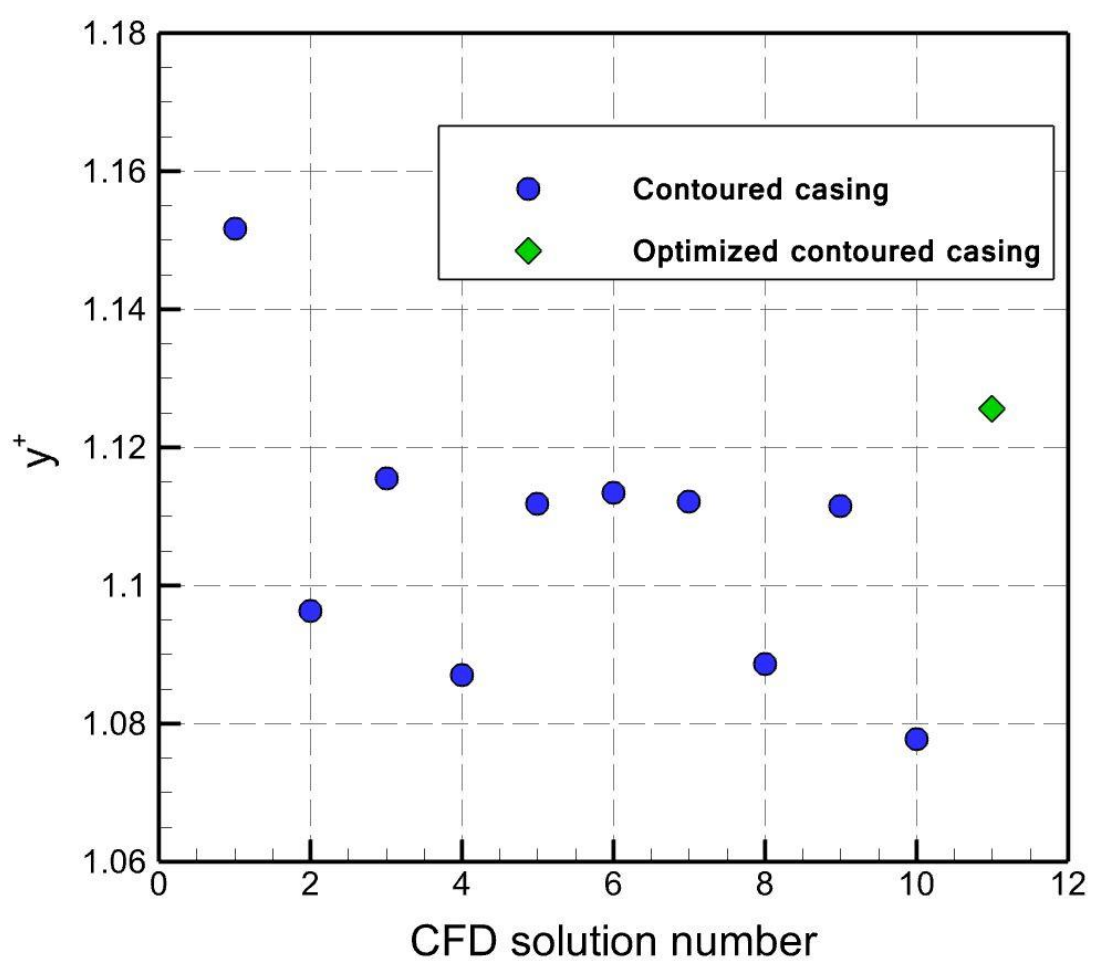

Fig. 3. Average value of $\mathbf{y}^{+}$over the casing, for different non-axisymmetric casing parameter values. 
The flow is modelled as dry air, under ideal gas and constant specific heat assumptions. The specific heat ratio $\gamma=1.4$, the specific gas constant $R=287 \mathrm{~J} \mathrm{~kg}-1 \mathrm{~K}-1$, and the dynamic viscosity is estimated by Sutherland's law. At the computational domain inlet, located 3.25 axial chords upstream of the stator blade leading edge, a total pressure type inlet boundary condition is used, by which the inflow is determined from the imposed stagnation flow properties. Inlet radial profiles of stagnation pressure, stagnation temperature, $k$ and $\omega$ were generated by an auxiliary computation by the EDDYBL program of Wilcox [29]. This program used the experimental inlet average total pressure and total temperature values to produce a fully developed inflow compressible boundary layer profile over the casing. The turbulence intensity as determined from the $k$ value at the passage mid-height is $5.15 \%$. This is typical of the relatively high level of turbulence in which turbine stages operate, at the rear end of a thermal cycle. At the computational domain outlet, located 2.54 axial chords downstream of the second stator blade trailing edge, the radial profile of static pressure measured in experiment is imposed and a static pressure outflow boundary condition is used. The hub, the casing, and the blade surfaces are modelled as no-slip adiabatic walls. Pitchwise periodic boundary conditions are imposed over the remaining boundaries. The rotor blades are stationary in the rotor frame of reference, which rotates at the constant rotor shaft speed stated in Table 3. The mixing plane formulation by Jasak and Beaudoin [30] is used $10 \mathrm{~mm}$ downstream the stator and rotor exit planes. 

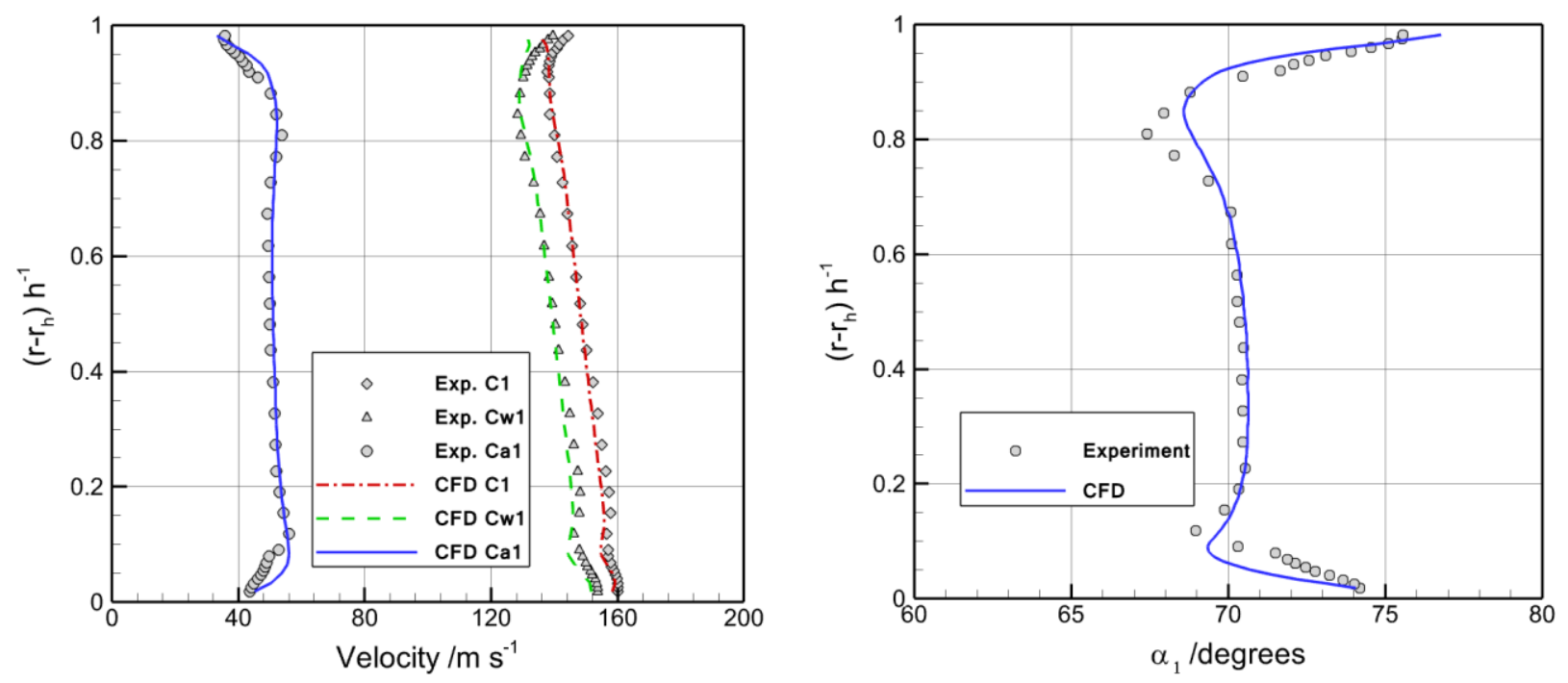

Fig. 4. Measured and calculated absolute (C1), axial (Ca1), and tangential (Cw1) velocity components and yaw angle at $8.8 \mathrm{~mm}$ behind the stator 1, pitchwise averaged.

The flow state was extracted from the CFD predictions at the same locations as in the experiment, these being 17 radial lines, with 38 points on each line. Fig. 4 shows the comparison between measured and calculated flow velocities and yaw angles $8.8 \mathrm{~mm}$ behind the first stator. Values are pitch averaged using a simple average. The CFD predictions appear to have captured the main trends in the experimental profiles, which indicates that the CFD model is adequate for guiding the end-wall design optimization, which is the main purpose of this study.

Fig. 5 (a) shows a flow visualisation over the stator 1 axisymmetric casing by near-surface limit streamlines. This visualisation indicates the presence of the flow structures outlined by Langston [4] that are often observed over turbine casing walls, as reported in the introduction. Specifically, the inflow to stator cascade blades features a growing boundary layer on the casing wall, along $143 \mathrm{~mm}$ long passage leading edge. The upstream boundary layer bifurcates at the leading edge of the blade, forming a saddle point. The location of this bifurcation is highlighted by the arrow in Fig. 5 (a). Due to the interaction of the end wall boundary layer and the adverse pressure gradient 
from the blade potential pressure field, a horseshoe vortex is generated near the junction between the blade leading edge and the end wall. The horseshoe vortex left and right arms bend downstream into the passage on both pressure and suction sides as shown by ribbons in Fig. 5 (b). The bundle of ribbons towards the right edge of Fig. 5 (b) show that the pressure side arm of the horseshoe vortex moves across towards the suction side, under the influence of a tangential pressure gradient, merging with the suction side at around 0.55 axial chord and creating a larger vortex structure, the passage vortex, as described by Langston [4]. The next section introduces a non-axisymmetric design of the stator casing to reduce the effect of these secondary flow features.

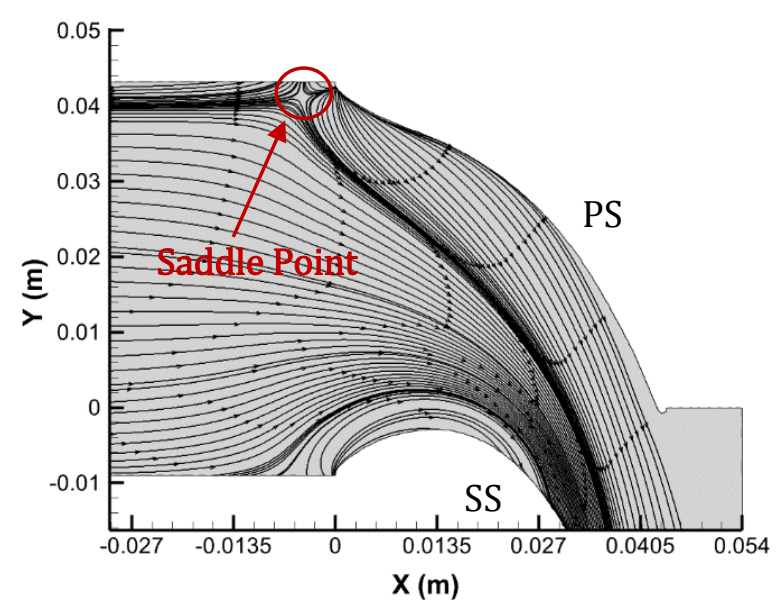

(a)

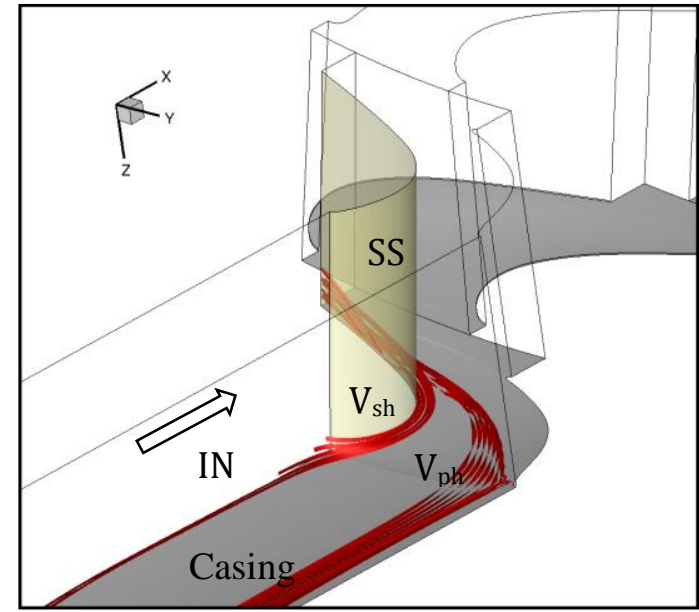

(b)

Fig. 5 (a) Flow visualization over the stator 1 axisymmetric casing showing the separation of oncoming casing boundary layer (saddle point) by the streamlines (b) The same surface showing by the ribbons the pressure side horseshoe vortex interacting with its suction side branch.

A 4760 cores High Performance Computer (HPC) cluster at the University of Leicester was used for the simulation, by domain decomposition. The Message Passing Interface (MPI) version of OpenFOAM was used as the flow solver, as its license is not core count limited. 


\subsection{Grid independence}

To investigate the influence of the spatial discretization on the flow predictions, a mesh convergence study is carried out using Richardson's extrapolation that is generalized by Roache [31]. In this work, three meshes are used to discretize stator 1 with a constant refinement ratio $r=$

2. These are a coarse mesh of $1.75 \mathrm{M}$ nodes (mesh 1), a mesh of intermediate spatial refinement of 3.5M nodes (mesh 2), and a fine mesh of $7 \mathrm{M}$ nodes (mesh 3). The grid convergence index (GCI) identifies to what extent the flow prediction approaches its asymptotic value and therefore gives an assessment of the appropriateness of the spatial resolution level. In the present work, the GCI is based on the averaged yaw angle predictions and the GCI is calculated according to Wilcox [32]. The average yaw angle predicted with each of the three meshes is $72.3^{\circ}, 71.6^{\circ}$ and $71.3^{\circ}$, respectively. The GCI computed from the average yaw angle predicted with the coarse mesh and that with the intermediate mesh is 0.916 . This is higher than the GCI computed from the average yaw angle predicted with the intermediate mesh and that with the fine mesh, which is 0.394 . This indicates a reduction in the mesh dependence in the numerical simulations in which the intermediate and the fine meshes are used. The difference between the yaw angles $\alpha_{2_{i}}$ predicted using the $i^{t h}$ mesh and the one obtained from Richardson's extrapolation $\alpha_{2_{R}}$ is used to define the relative error as $\epsilon_{i}=\alpha_{2 i} \alpha_{2}^{-1}{ }_{R}-1$.

Fig. 6 shows the relative error based on Richardson's extrapolation with the mesh refinement level, which is defined as the ratio of the number of nodes on the $i^{\text {th }}$ mesh to the number of nodes of the coarse mesh. As the mesh is refined, the difference between the simulation and the extrapolated value becomes small. The magnitudes of the relative errors reported in Fig. 6 are $\epsilon_{1}=$ $1.72 \%, \epsilon_{2}=0.73 \%$, and $\epsilon_{3}=0.31 \%$. As the difference in the relative errors between the intermediate mesh and the fine mesh is below $1 \%$, the mesh of intermediate spatial resolution is 
selected for the current study.

The total pressure loss coefficient through the stator, as defined by Equation (1) of Section 5, was determined from the flow predictions with the coarse, intermediate, and fine meshes. Increasing the spatial resolution changes the stator total pressure loss coefficient from 0.0561 , to 0.0570 and to 0.0573 . The difference between the intermediate and fine mesh prediction is 0.0003 and it will be shown in Section 6 to be one order of magnitude lower than the total pressure loss coefficient reduction obtained by using a contoured casing.

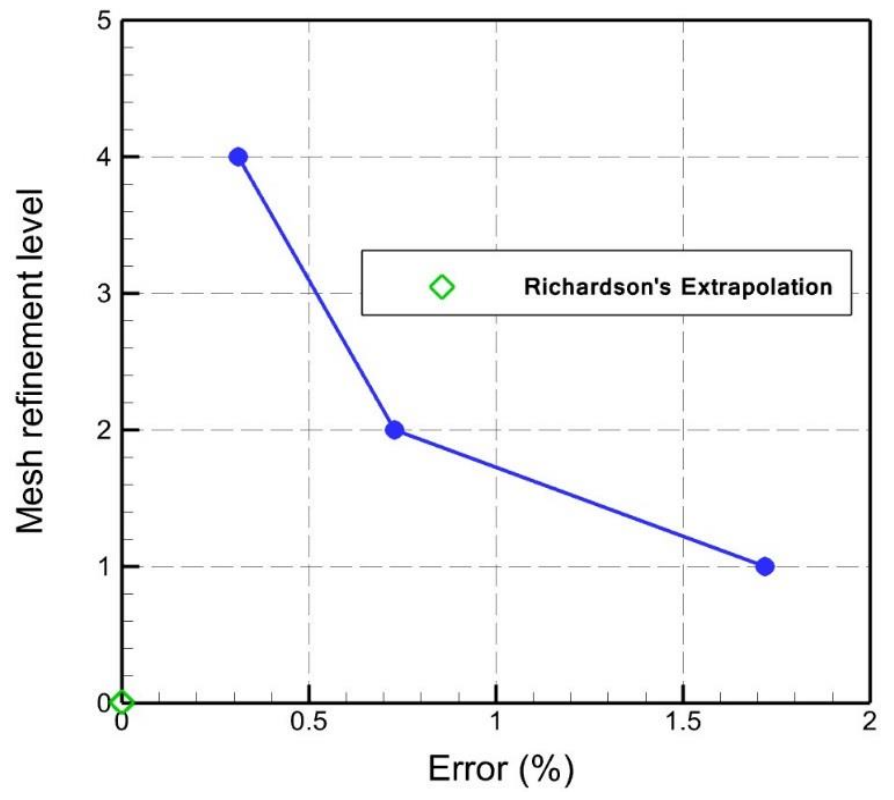

Fig. 6. Error reduction with increasing spatial resolution based on Richardson's extrapolation.

\section{Non-Axisymmetric Upstream Stator Casing Design}

The non-axisymmetric casing design workflow has been implemented in the Alstom Process and Optimization Workbench (APOW) software, as shown in Fig. 11. The casing wall is contoured by applying a groove to it, while the hub is kept axisymmetric. The RWTH Aachen dataset of points $P_{i}$ in $(x, r, \theta)$ is re-stated in $3 \mathrm{D}$ Cartesian coordinates as $P_{i}=\left(x_{i}, r_{t} \cos \theta_{i}, r_{t} \sin \theta_{i}\right)$ for compatibility with ANSYS ICEM CFD. 
Three surfaces were modelled mathematically to represent the upstream stator casing delimiting one flow passage. These are referred to as the blade to blade passage surface, the extended inlet surface, and the extended outlet surface. 65 points define the blade pressure side and 47 points the blade suction side. These points were projected on the casing cascade plane in MATLAB by the function $P_{i}=\left[x_{i}, \frac{\pi}{2}-\arctan \left(\frac{y_{i}}{z_{i}}\right)\right]$.

The projected points were interpolated using smoothing cubic splines by the MATLAB functions csaps and ppval. This gave the cylindrical casing surface shown in Fig. 7 defined as the generalized surface $s(u, v)=\left[u, r_{t} \cos (v), r_{t} \sin (v)\right] . s(u, v)$ was re-stated in MATLAB as a non-uniform rational B-spline (NURBS) surface. For this purpose, two open uniform knot vectors were used. The number of knots $m+1$ and of the control points $n+1$ on the NURBS are related as $m=n+k$, where $k$ is order of the NURBS surface which is restricted to 3 in ICEM CFD. The internal knot values are equally spaced and identified using the parametrization weight according to Piegl and Tiller [33]. Three NURBS surfaces were generated and exported as IGES format files, which is a supported input file format of ICEM CFD. By using NURBS surfaces, the cylindrical casing was represented as an exact geometry.

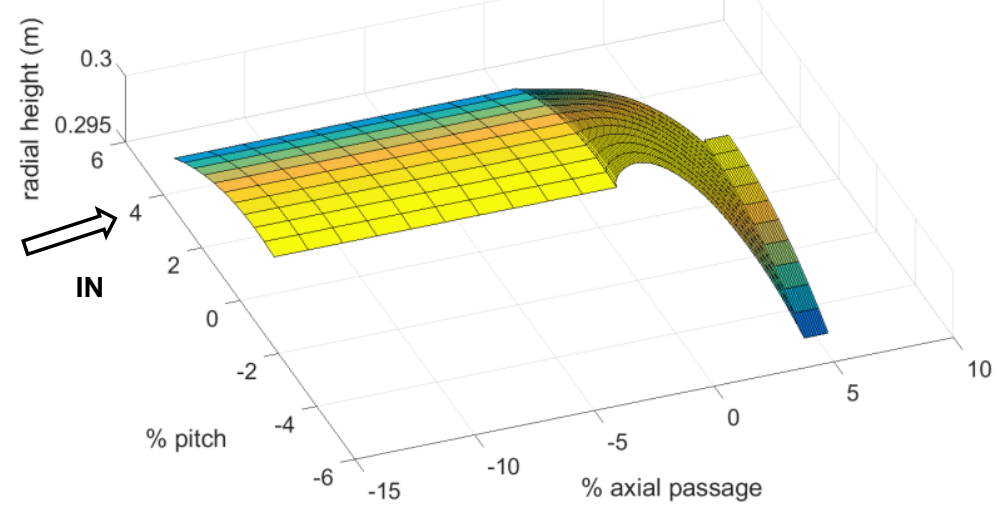

Fig. 7. Casing surface rendered as a three sets of $\boldsymbol{s}(\boldsymbol{u}, \boldsymbol{v})$. 
The next step is to define a grooved casing surface. The path of the groove shown in Fig. 8 is defined based on the profiles of turbine blades, inflated in the cascade plane. This is achieved by offsetting the blade perimeter by a set distance, normal to the blade perimeter. The groove path $\xi^{a}\left(x, \theta_{g}\right)$ is then defined by linear interpolation between the inflated profiles. For each point $P(x, \theta)$ on the casing plane $r=r_{t}$, its normal distance to the groove path is $\eta(x, \theta)=$ $\left(\left\|\xi^{a}(x)-P(x, \theta)\right\|\right)$

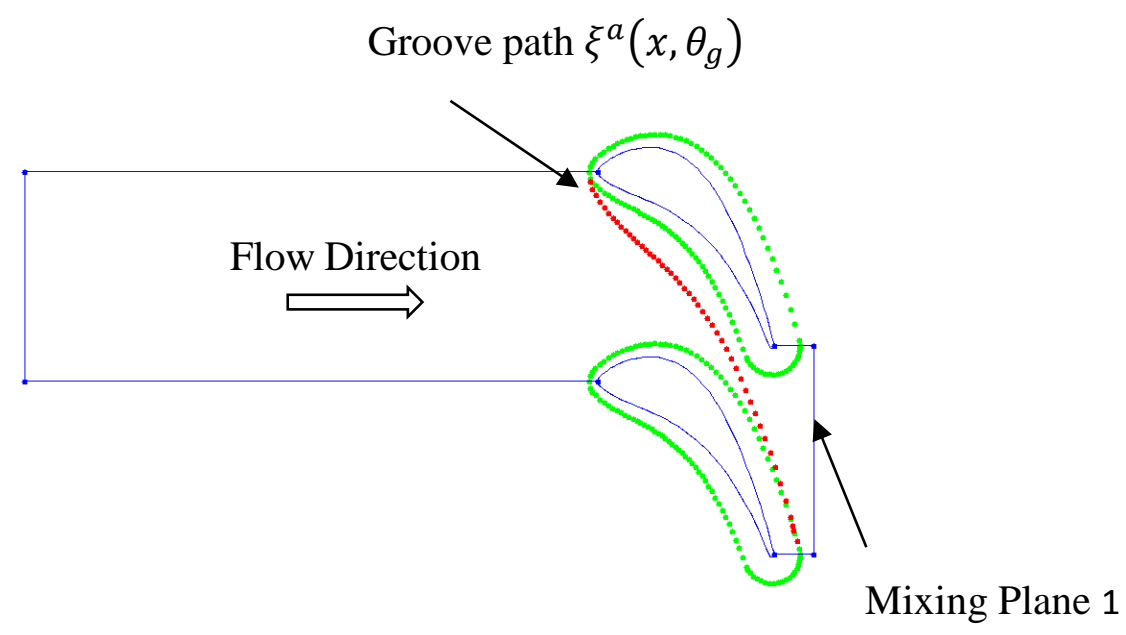

Fig. 8. Stator blade profiles 'inflated' in the annular cascade casing plane (green) and interpolated groove path (red).

The groove depth $g(d, \eta)=h_{o} d^{-4}\left(\eta^{2}-d^{2}\right)^{2}$, where $d$ is a set angle in radians from the curve path. The groove width $w=2 d$ varies along the groove path $\xi^{a}\left(x, \theta_{g}\right)$ as a user-defined free parameter. The groove path starts from upstream of the leading edge and ends at the stator 1 to rotor mixing plane. The mid-width groove depth $h_{o}\left(\xi^{a}\right)$ along the groove path $\xi^{a}\left(x, \theta_{g}\right)$ is defined by the Beta distribution function (c.f. Devore [34]) $f(s, \alpha, \beta)=\Gamma(\alpha+\beta) \Gamma^{-1}(\beta) \Gamma^{-1}(\alpha) \theta^{\alpha-1}(1-$ $\mathrm{s})^{\beta-1}, 0<\mathrm{s}<1$. The maximum groove depth is $3 \mathrm{~mm}$ located at $\xi^{a}=\mu$, where $\mu$ is userdefined. From this, the stator 1 casing radius is defined as $r_{t g}(x, \theta)=$ 
$\left\{\begin{array}{ll}r_{t} & |\eta|>d \\ r_{t}+g[d, \eta(x, \theta)], & |\eta| \leq d\end{array}\right\}$ and the stator 1 casing surface is defined as $s(u, v)=$ $\left[u, r_{t g}(u, v) \cos (v), r_{t g}(u, v) \sin (v)\right]$ as shown in Fig. 9.

The Beta distribution was used as it avoids by construction any mismatch in the surface radial height at the outer perimeter of the grooved area [35]. It therefore improves the surface transition between the grooved area and the remainder of the casing compared to using a Gaussian distribution as in Reutter et al. [36]. This surface transition is explained in further details in Kadhim et al. [35].

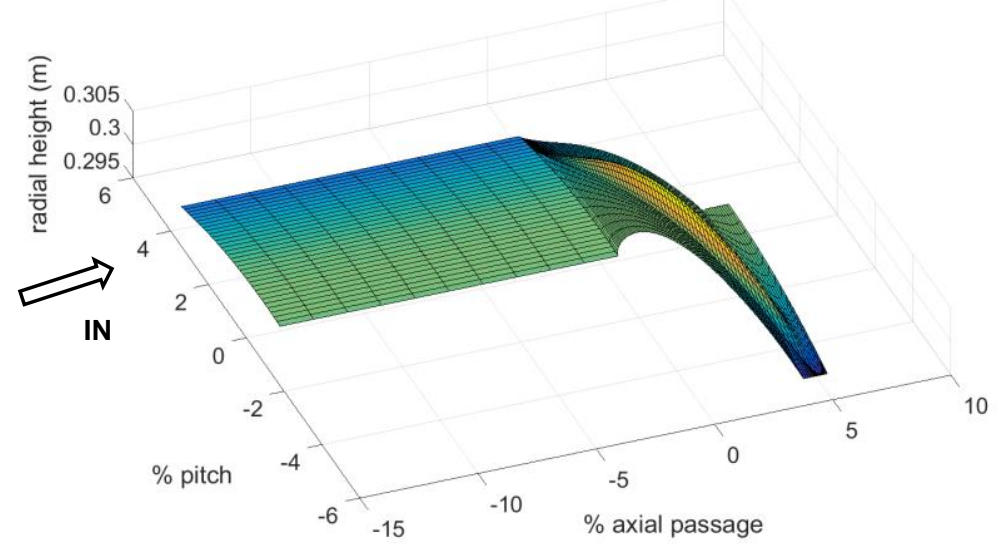

Fig. 9. Casing surface rendered as a three sets of $\boldsymbol{s}(\boldsymbol{u}, \boldsymbol{v})$, including a diffusing groove.

The $s(u, v)$ was re-stated as a NURBS surface, mated with the extended inlet surface and the extended outlet surface as shown Fig. 10 and exported as an IGES file to ICEM CFD. 


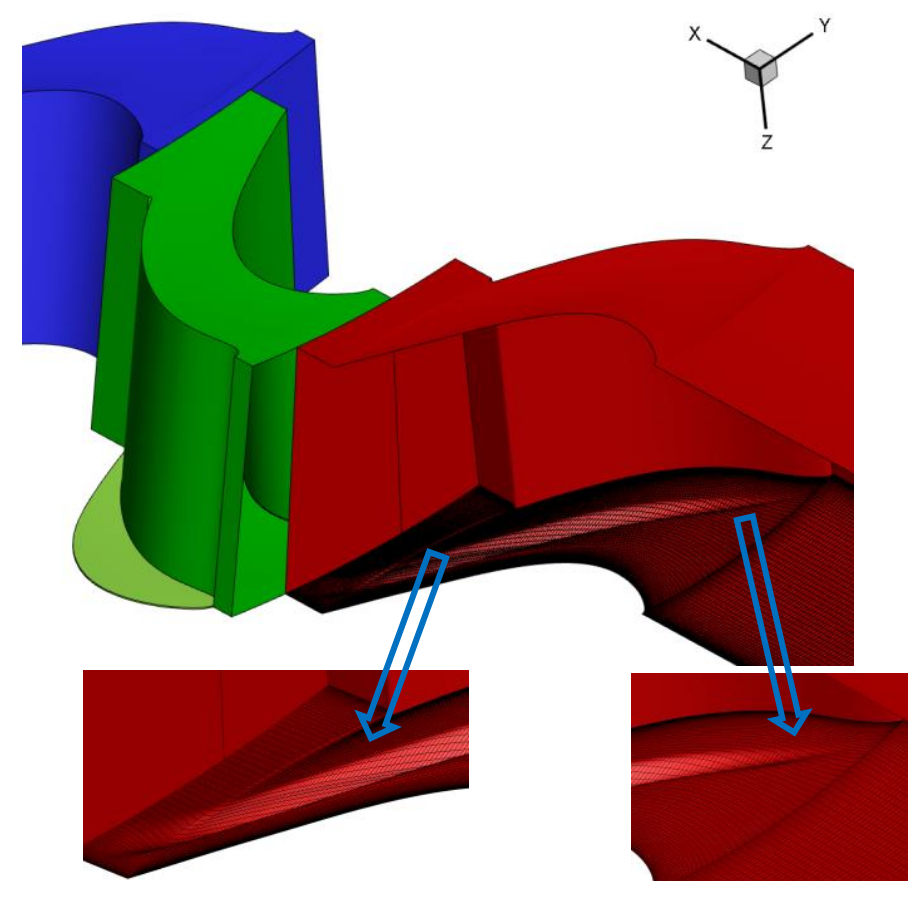

Fig. 10. Non-asymmetric casing NURBS imported in ANSYS ICEM CFD.

\section{Contoured Casing Optimization}

The contoured end wall definition of the previous section uses a small set of parameters to make the optimization process by APOW more treatable. Three main variables were used for the optimization process: the maximum groove depth location along the groove path $\mu\left(x, \theta_{g}\right)$, the groove width at the blade leading edge $d_{L E}$ and the groove width at the blade trailing edge $d_{T E}$. The groove width at the blade leading edge $d_{L E}$ was found to have a small effect on the total pressure loss, therefore, this parameter is optimized segregated and last. The total pressure loss coefficient $C_{P t}$ was used as the objective function. This was implemented in two different ways. Firstly, the objective function is the stator row total pressure loss coefficient

$$
C_{P t r}=\frac{P_{t o}-P_{t 1}}{P_{t 1}-P_{1}}
$$


The second objective function is the stage total pressure loss coefficient

$$
C_{P t s}=\frac{P_{t o}-P_{t 2}}{P_{t 2}-P_{2}}
$$

where subscript 0 represent stator inlet plane, 1 represent stator exit plane and 2 represent the rotor exit plane. The contoured casing surface generated in MATLAB and imported as NURBS in ICEM CFD delimits the computational domain. This was discretized in ICEM CFD maintaining similar meshing parameters to obtain the same mesh quality as for the validation test case. The unstructured mesh was translated to OpenFOAM. The same boundary conditions were applied as for the validation test case and the numerical solution was iterated by OpenFOAM convergence, as assessed by the reduction in the residuals by $10^{-6}$ with respect to their values at the start of the computation.

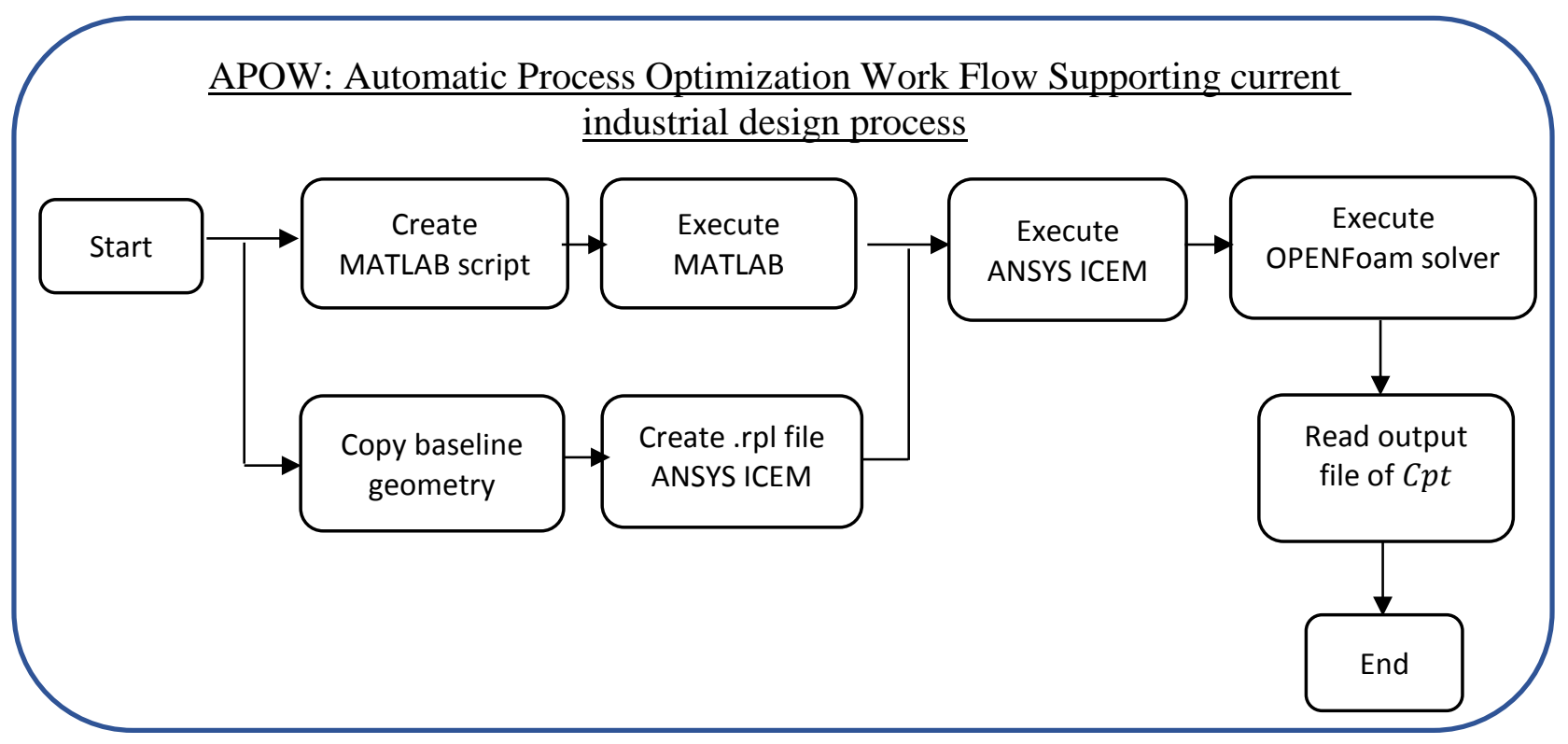

Fig. 11. Casing endwall design workflow.

The workflow of Fig. 11 was executed in batch mode for different values of $d_{L E}, d_{T E}$, and $\mu\left(x, \theta_{g}\right)$ using APOW. The APOW Design of Experiments (DOE) was used to generate the design 
space, based on the Optimal Latin Hypercube Design technique. The optimization analysis of DOE was performed on a surrogate model. For this purpose, Kriging was selected as the surrogate model type. There is a growing body of literature that documents the use of Kriging for generating reduced-order models for optimizing the design of axial turbines. Alternative approaches include genetic algorithms, steepest descent algorithms, and adjoint methods. In this work, Kriging was used as it can give a good compromise between the computational cost and the prediction accuracy. It allows to properly capture the complexity of a response surface featuring sharp curvature changes, even in the presence of highly irregular distributions of interpolation sampling points [37]. The percentage difference between the optimal total pressure loss prediction from Kriging and from CFD is 1.5 , indicating a good model data fit. For more details on DOE surrogate modelling techniques can be found in [38].
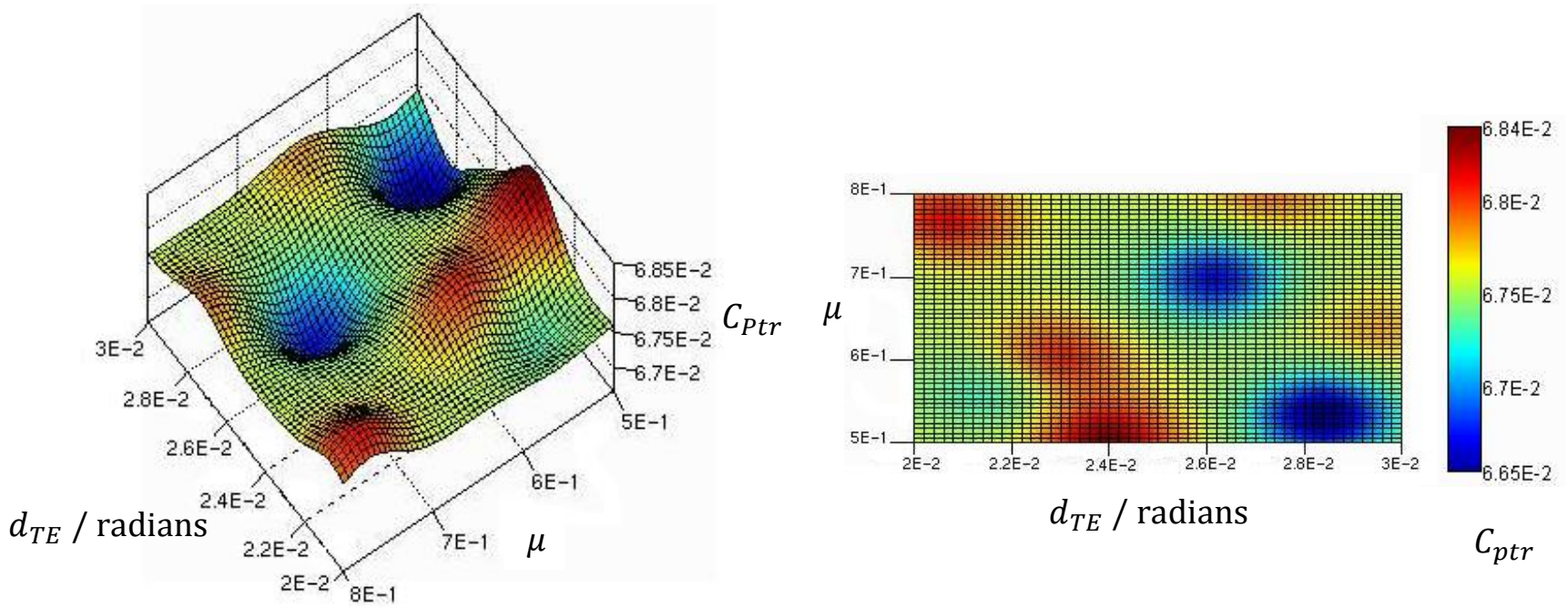

Fig. 12. Optimization of the casing groove using surrogate modelling and Kriging.

Fig. 12 shows a sample response function of the optimization method, stated in terms of the stator row total pressure loss coefficient. The changes in the response function are studied for different values of the groove width at the trailing edge, $d_{T E}$, and the axial location of maximum 
groove depth, $\mu$. Kriging provides a continuous response function from a discrete sample of computational fluid dynamics estimates of the total pressure loss, it enables to develop an insight of how the design parameters $d_{T E}$ and $\mu$ influence the performance of the turbine across the full extent of the parameter space and regions of high performance, with acceptable sensitivity. From the Kriging results, a region of low $C_{P t}$ was identified over the range 0.02 radians $\leq d_{T E} \leq 0.03$ radians and $0.5 \leq \mu \leq 0.8$. It is of interest to explore the flow features characterizing this parameter space, which are explored in the next section. The optimum values of the design variables were selected corresponding to the minimum value of $C_{P t}$ shown in Fig. 12.

The final optimized design uses a casing with a narrow groove at the leading edge, of width $d_{L E}$ $=0.005$ radians. The groove expands sideways along its length up to the trailing edge, where the groove width is $d_{L E}=0.285$ radians. The maximum groove depth $\mu$ is located at $80 \%$ of the axial chord. The groove path is as defined in section 4 and is shown in Fig. 8. This final optimized design is shown in Fig. 13.

For each optimization process, 15 individual CFD solutions were used for constructing the response function, with Kriging. Each CFD solution typically took 10 hours of computer wall time on 50 HPC cores. The wall time used for obtaining each response function was about 150 hours, as the CFD solutions were run sequentially, due to constraints on the available computational resources. Running the solutions in parallel would have reduced the wall time to 10 hours.

\section{Flow Analysis}

The validated simulation of the Aachen Turbine flow passage was used as the baseline for studying the effects of contouring the casing, using Tecplot 2015 as the CFD post-processor. Fig. 13 shows the flow over the APOW optimized casing by ribbons, which illustrate the vorticity along 
limit streamlines by the twists in them. The blade is oriented with the blade height increasing towards the floor of the image, as highlighted by the Cartesian axes inset. The ribbons mark the path of the horseshoe vortex suction and pressure side branches by their twisted segments. This path is highlighted in the enlargement of Fig. 13 (b), where the casing is viewed from the leading edge. The pressure side branch of the horseshoe vortex, guided by the groove, approaches the blade suction side at about $x / c=0.69$. Its interaction with the suction side branch is delayed towards the passage trailing edge, as shown in the magnification of Fig. 13 (c) where the casing is viewed from the stator 1 trailing edge plane. This delay typically reduces the passage vortex size, the extent of the main passage blockage from this secondary flow feature, and hence the blade and stage pressure loss coefficient, as confirmed in Table 2 .

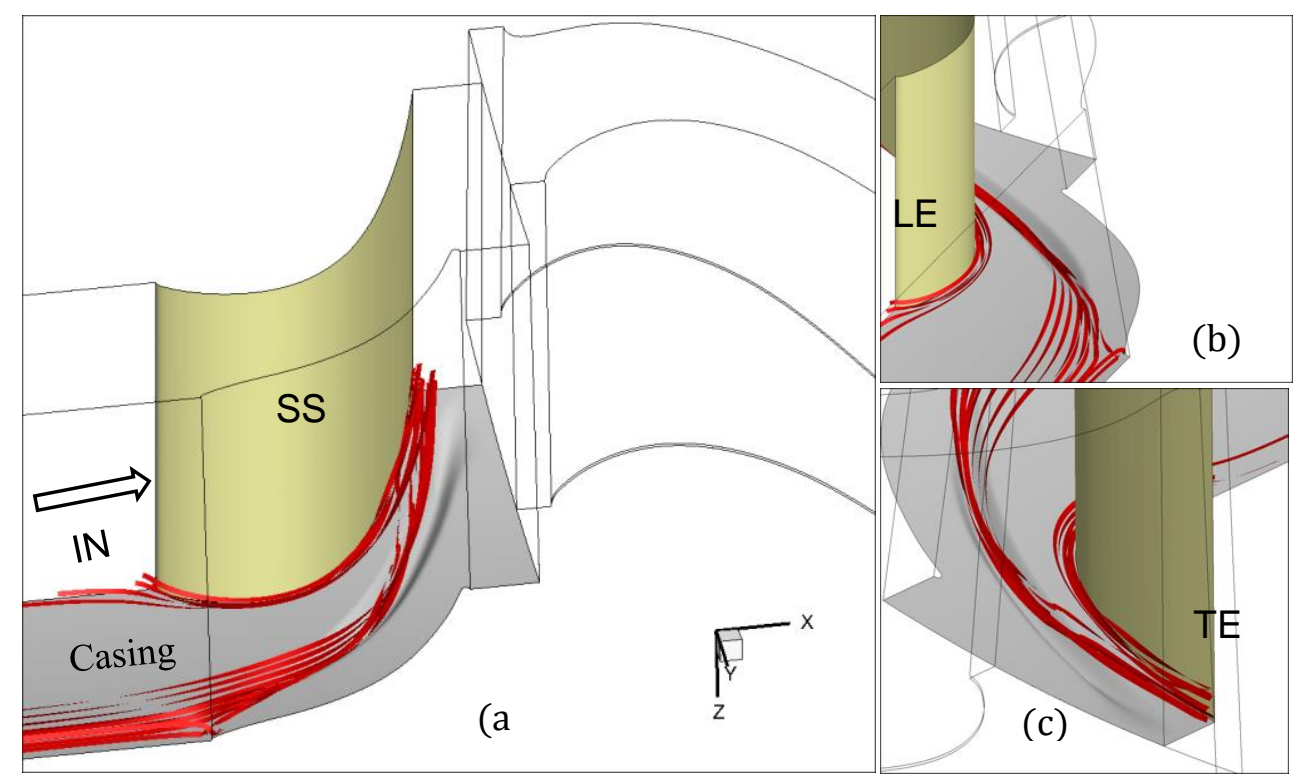

Fig. 13. Prediction of the flow near the grooved casing visualized by ribbons. (a) blade passage, (b) view from the leading edge, and (c) view from the trailing edge. 


\section{Table 2}

Baseline and optimized Aachen Turbine performance.

\begin{tabular}{|c|c|c|c|c|c|c|}
\hline \multirow{2}{*}{$\begin{array}{c}\text { Parameter } \\
\begin{array}{c}\text { Total pressure loss } \\
\text { coefficient }\end{array}\end{array}$} & \multicolumn{3}{|c|}{ Stator 1 plane } & \multicolumn{3}{|c|}{ Rotor plane } \\
\hline & Baseline & Contol & $\mathrm{d} \Delta \%$ & Baseline & Contoured & $\Delta \%$ \\
\hline Design conditions & 0.0670 & 0.0644 & -3.881 & 9.670 & 9.579 & -0.941 \\
\hline Off design $(72 \% \dot{\theta})$ & 0.0764 & 0.0733 & -4.058 & 11.889 & 11.766 & -1.035 \\
\hline
\end{tabular}

Fig. 14 (a-d) show the mid-span to casing radial distributions of the pitch-averaged total pressure loss coefficient $C_{p t r}$ and yaw angle $\alpha_{1}$, at the stator 1 exit plane. $C_{p t r}$ and $\alpha_{1}$ are compared between the baseline and the optimized casing simulations. The yaw angle distribution in Fig. 14 (a) is mostly above the $70^{\circ}$ design point. This results in overturning near the casing. The reason behind this, is the effect of the cross-flow pressure gradient on the casing boundary layer, which more easily turns the less energetic flow compared to the mid-passage. By contouring the casing, Fig. 14 (a) shows that this overturning is reduced, leading to a more spanwise uniform $\alpha_{1}$. This change is associated to a reduction in total pressure loss coefficient, as shown in Fig. 14 (b).

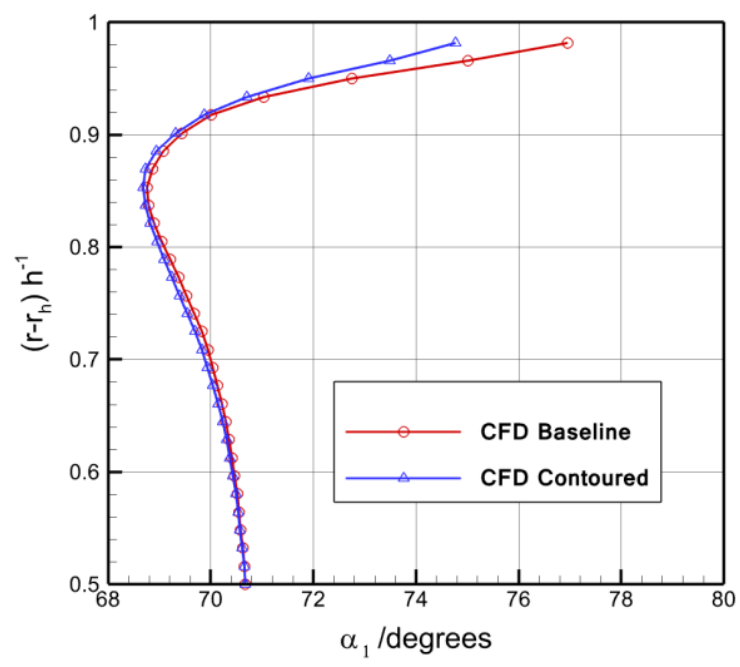

(a)

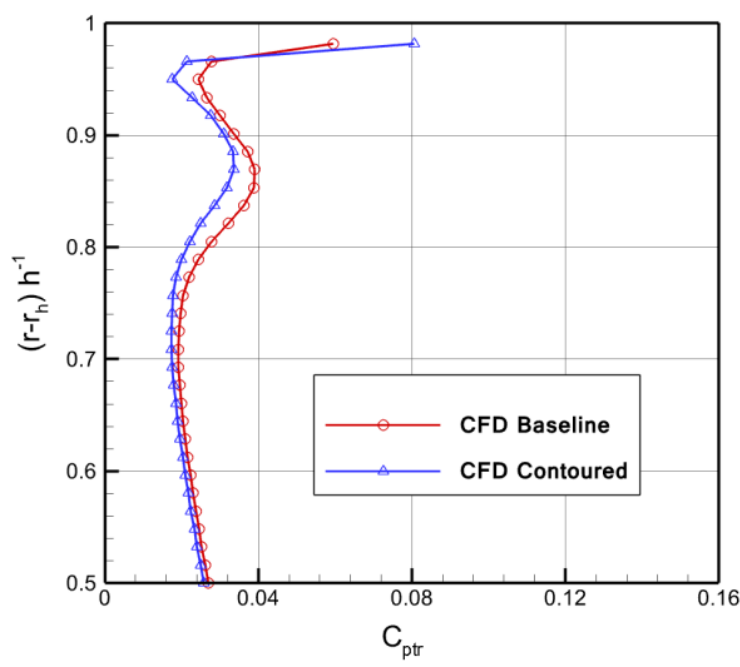

(b) 


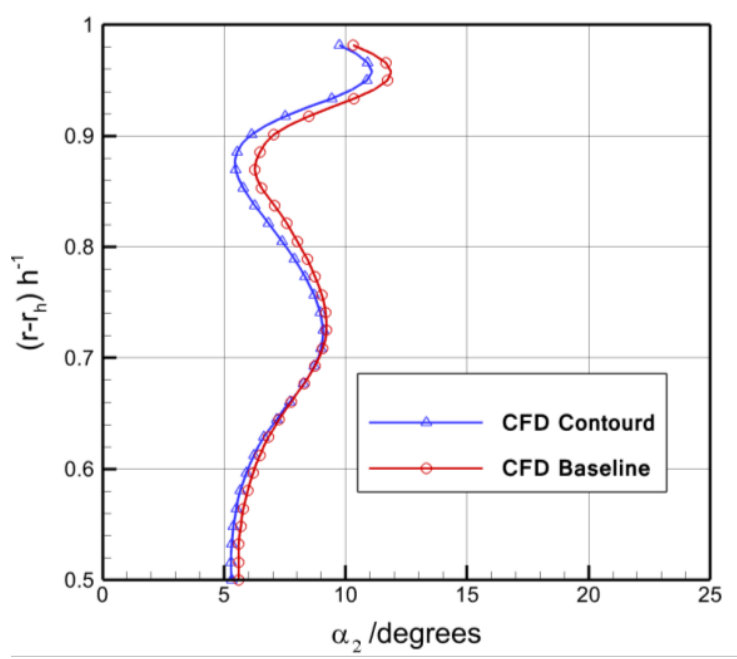

(c)

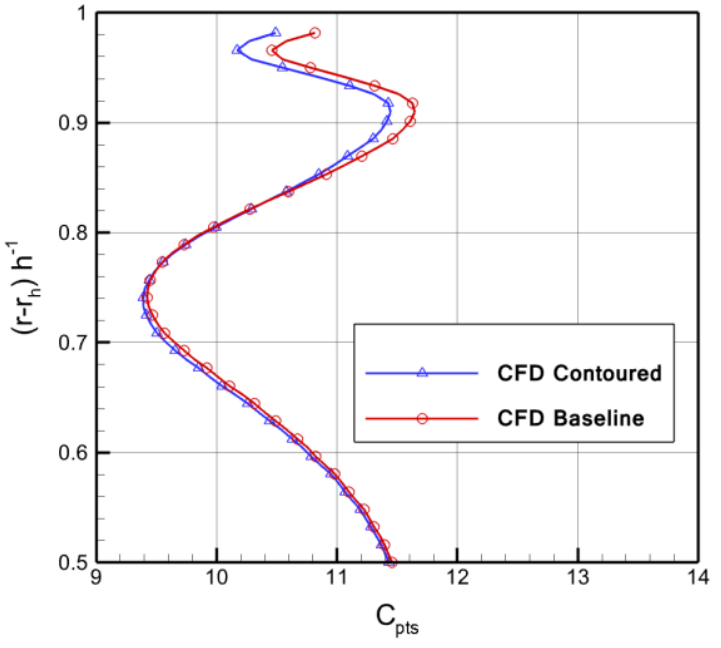

(d)

Fig. 14. Predicted pitch-averaged total pressure loss coefficients and yaw angles along the normalized span. $(a, b)$ stator 1 plane, $(c, d)$ rotor plane.

The results downstream of the rotor are evaluated in the plane 2 of Fig. 14, $8.8 \mathrm{~mm}$ behind the rotor trailing edge. The reduction in the overturning at the casing behind the stator 1 leads to a small reduction in the pitch-averaged yaw angle at the rotor exit, mainly towards the casing, as shown in Fig. 14 (c). As a result, the pitch-averaged yaw angle distribution, $\alpha_{2}$ appears to be more uniform with the contoured casing, below 0.9 blade span. The total pressure loss coefficient at the rotor exit, shown in Fig. 14 (d), indicates an improved end-wall flow, from a reduction in $C_{p t s}$ mainly towards the casing. This the location of the reduction in total pressure loss coefficient close to the casing suggests a possible reduction in the rotor tip leakage loss. These changes lead to a small reduction in the passage total pressure loss coefficient, as reported in Table 2. 


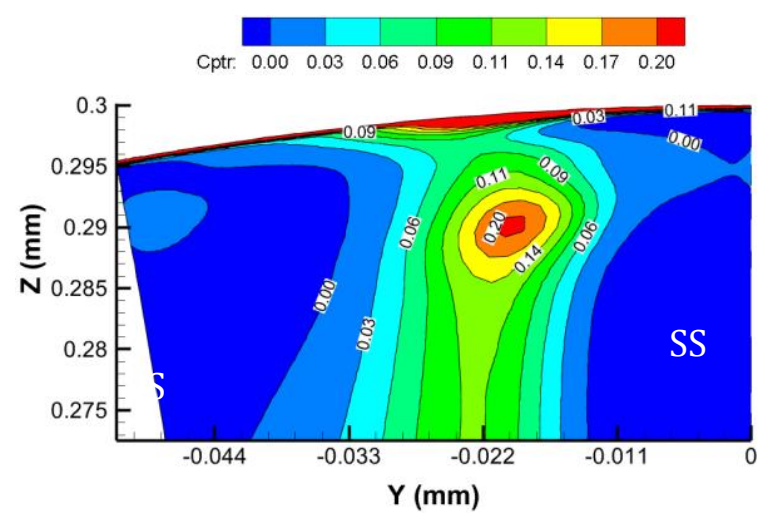

(a)

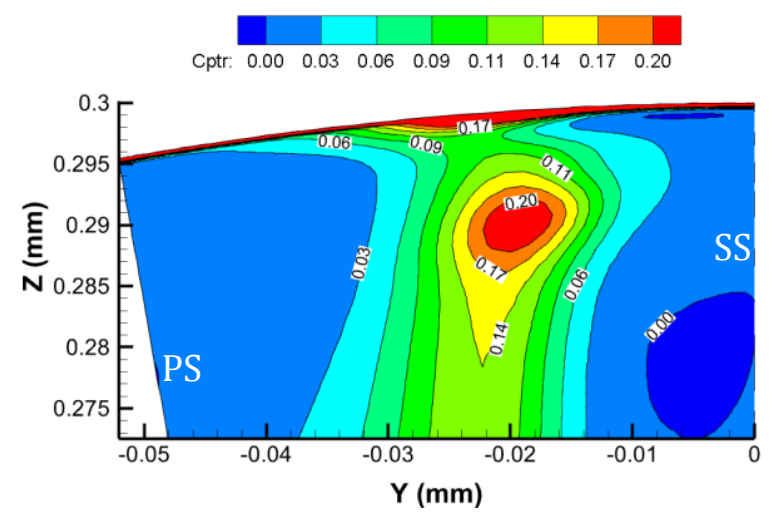

(c)

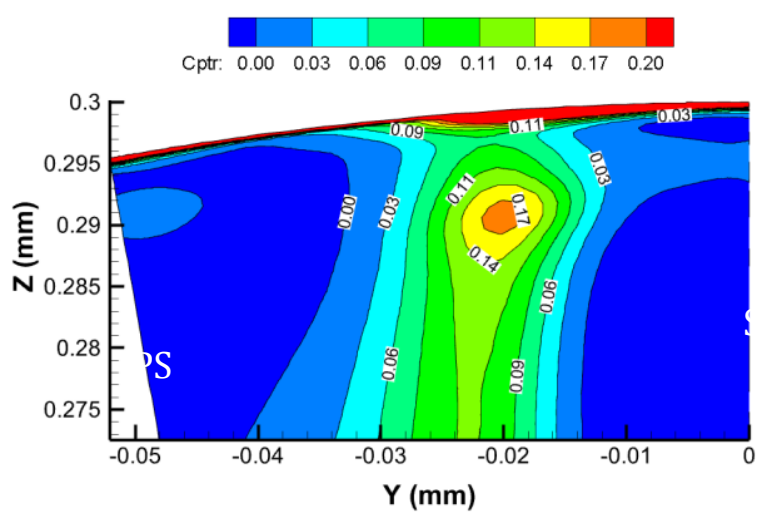

(b)

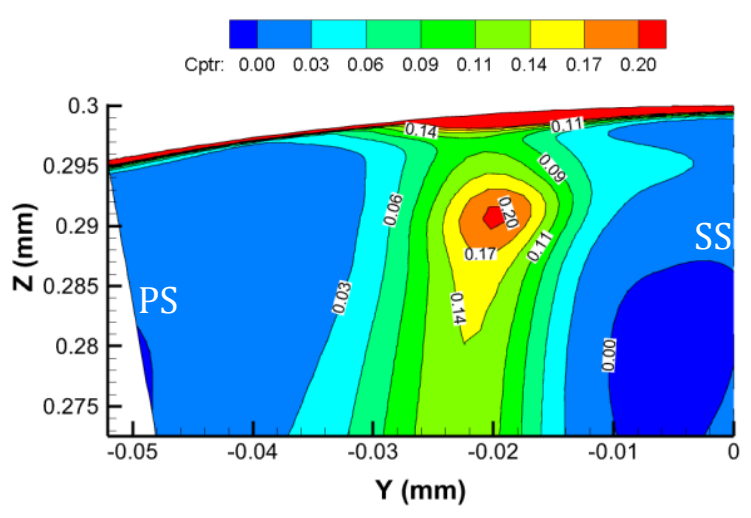

(d)

Fig. 15. Contours of total pressure loss coefficient at $8.8 \mathrm{~mm}$ behind the stator 1 at the axial stage design point (a, b) and off design (c, d); (a, c) axisymmetric casing, (b, d) contoured casing.

Table 3 shows the design and off design operating conditions modelled by CFD. The nonaxisymmetric casing design, optimized at the design point, was tested numerically off-design by reducing the rotor speed to 2510 r.p.m. This set point operation was reported by Gallus and Zeschky [39] as lowering the turbine isentropic efficiency due to higher secondary flow losses. Table 2 shows that off-design the new casing geometry provides a total pressure loss reduction comparable to that at the design rotor speed. The isentropic stage efficiency off-design increased by $0.73 \%$ compared to an increase of $0.69 \%$ at the design rotor speed, due to the higher secondary flow losses that are mitigated off-design. Therefore, performance gains are obtained both at design 
and off-design.

\section{Table 3}

Summary of Aachen Turbine operating conditions modelled by CFD.

\begin{tabular}{c|r|c}
\hline \hline Case & Rotational speed & Mass flow rate \\
\hline Design point & $3500 \mathrm{rpm}$ & $6.7 \mathrm{~kg} / \mathrm{s}$ \\
Off design & $2500 \mathrm{rpm}$ & $6.7 \mathrm{~kg} / \mathrm{s}$ \\
\hline \hline
\end{tabular}

An insight into the through-flow at design and off-design conditions is provided by Fig. 15, which presents the contours of total pressure loss coefficient at $8.8 \mathrm{~mm}$ behind the first stator with an axisymmetric a contoured casing. Fig. $15(\mathrm{a}, \mathrm{b})$ shows the contours of $C_{P t}$ at the axial turbine stage design point, while Fig. $15(\mathrm{c}, \mathrm{d})$ shows the contours of $C_{P t}$ off design. Fig. 15 (a-d) show a core of high total pressure loss coefficient, located approximately at $y=0.291 \mathrm{~mm}$ and $z=-0.019$ $\mathrm{mm}$. The location of this maximum is consistent with that of the casing passage vortex (PV) in [26]. By contouring the casing, the extent of this high total pressure loss coefficient area is reduced in Fig. 15 (b) compared to Fig. 15 (a). A similar reduction in the total pressure loss coefficient maximum, associated with the casing passage vortex, is noticeable at the off-design condition of Fig. 15 (d) compared to Fig. 15 (c), for which an axisymmetric casing is used. These results indicate that the authors' contouring is effective in reducing the passage vortex associated loss at design and off design point operations. 

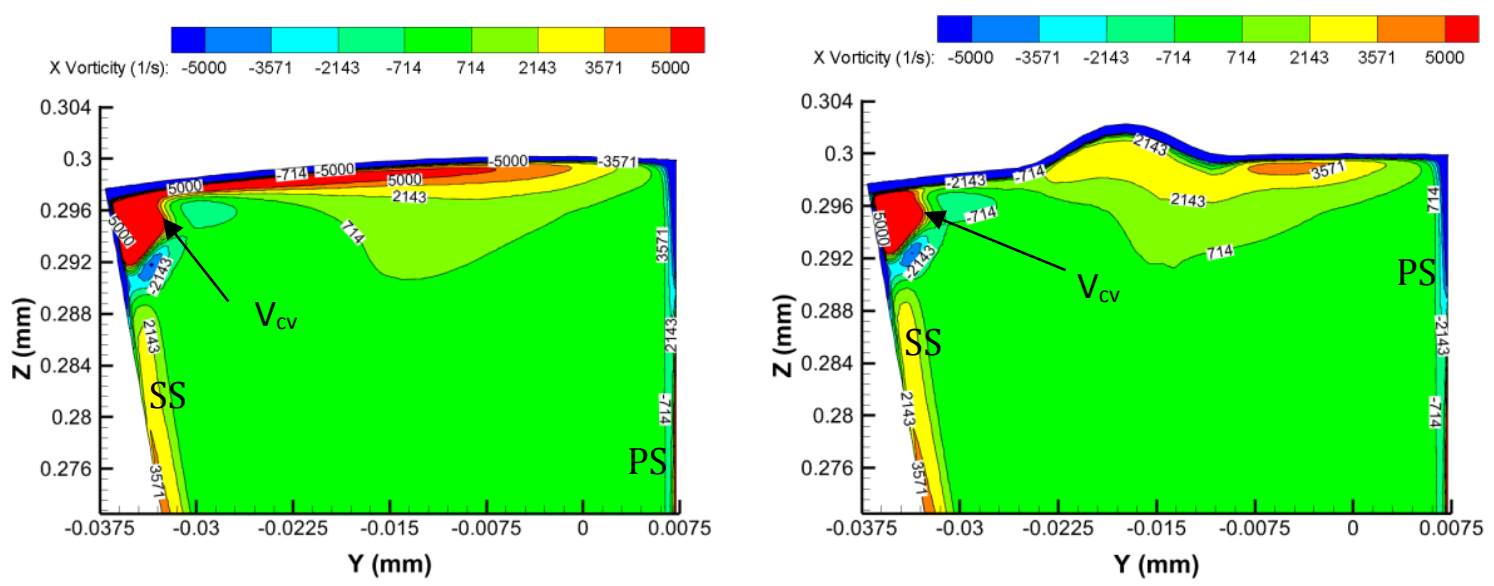

Fig. 16. Contours of axial vorticity through stator 1 at 0.8 axial chords. Design point.

A further insight into the effects of contouring the stator casing wall on the passage flow is provided by Fig. 16, which shows contours of axial vorticity at $x=0.8 C_{x}$ at the design point with and without a contoured casing. From Fig. 16, contouring reduces the axial vorticity magnitude over the casing wall, which is indicative of a lower strain rate in the casing boundary layer. This would be consistent with a reduction of the passage cross-flow, from the pressure side to the suction side, that sees a longer and more tortuous path over the contoured casing than over the axisymmetric casing. Contouring also appears to be beneficial to the interaction between the suction side corner vortex, labelled as $\mathrm{V}_{\mathrm{cv}}$ in Fig. 16, and the end-wall flow. With an axisymmetric wall, same sign axial vorticity from the casing appear to feed the corner vortex. With the contoured wall, this process is essentially removed. This may result in a lower streamwise growth rate of the corner vortex. This confirms that the presence of the groove has changed the secondary flow structure in the end-wall region. Off-design, a similar pattern is obtained as in Fig. 16. 


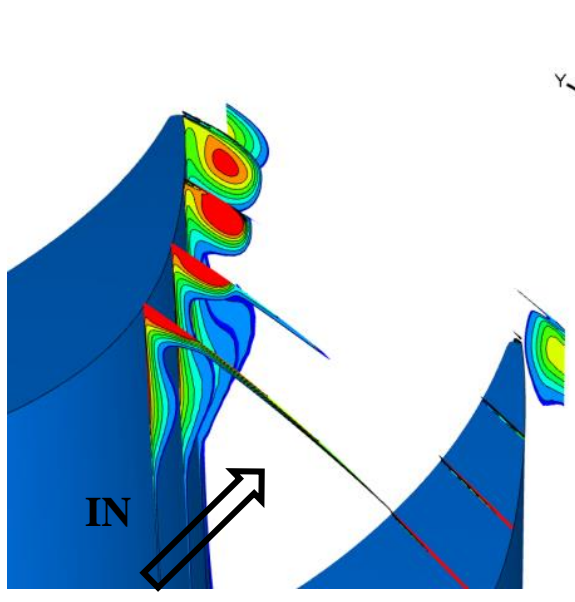

(a)

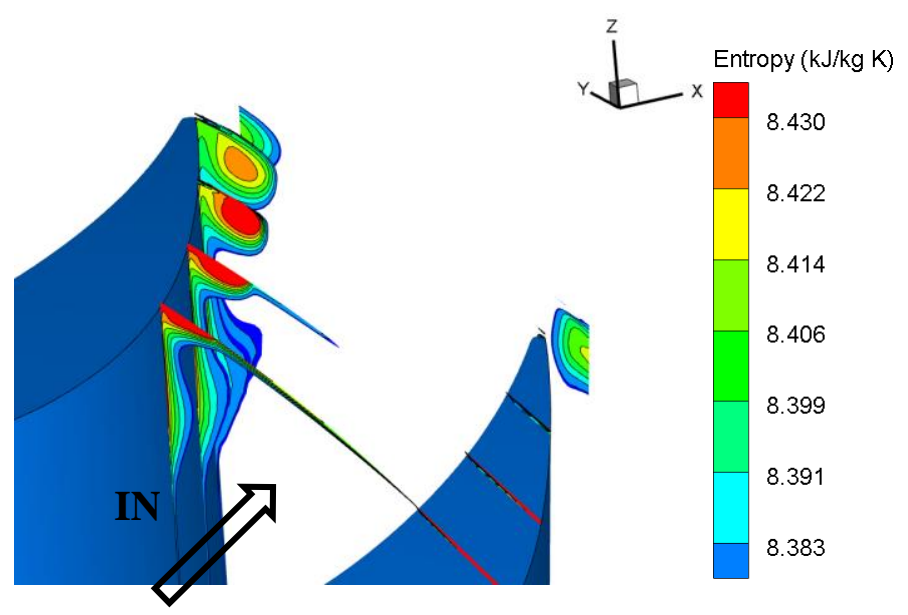

(b)

Fig. 17. Entropy distributions at different axial positions for the rotor passage at the axial stage design: (a) axisymmetric casing, (b) contoured casing.

The 3-D flow inside the rotor passage is dominated by the interaction of secondary flows from stator 1 with the passage vortex and the tip leakage vortex of the unshrouded rotor blade. Fig. 17 shows the entropy distributions across different axial planes through the rotor passage with and without the contoured casing. It can be seen that the entropy 'core' that forms near the blade suction side tip reduces in peak value and in cross-section in Fig. 17 (b) compared to Fig. 17 (a). This is likely to indicate a reduction in the tip leakage vortex strength that in turns reduces the flow overturning, as shown in Fig. 14 (c).

\section{Conclusions}

A new casing end-wall surface definition was applied to optimize the aerodynamic performance of a 1.5 stage axial turbine. Measurements from RWTH Aachen were used to validate an OpenFOAM 3.2 extend steady 3D $k-\omega$ SST model of the Aachen Turbine and gave confidence in its use for studying the effect of the new casing on the flow. This approach for designing the casing wall uses a guide groove elicited from the natural path of the end-wall secondary flows. A 
set of parametric equations is used with the Beta distribution function to design the casing groove path, which is a first application of the Beta distribution function to the contouring of a turbomachine casing. This design was optimized using Alstom Process and Optimization Workbench (APOW) software. The current design approach for a stator turbine casing is shown to produce aerodynamic performance gains both at design and off design. There appears to have been a positive effect on the rotor tip leakage flow.

Numerical flow visualizations appear to support the underlying principle of the nonaxisymmetric casing design, namely that the groove guides the pressure-side horseshoe vortex branch, delaying the passage vortex formation. The optimal design was obtained by a computerdriven process that appeals to the industrial design process of turbomachines due to its autonomy, as it required modest user intervention once set up. The industry-wide adoption of this technology would have significant economic and environmental impacts.

\section{Acknowledgement}

This work was undertaken under the auspices of the GE \& Alstom Energy - University of Leicester framework agreement. Advice from Dr. N. Z. Ince and Dr. M. Willetts, GE, is gratefully acknowledged. The Higher Committee for Education Development in Iraq (HCED), is acknowledged. This research used the ALICE high performance computing facility at the University of Leicester. Graphical rendering software licenses were originally acquired with EPSRC support on Grant GR/N23745/01. The supply of experimental data for the 1.5 stage axial flow turbine "Aachen turbine" under license by RWTH Aachen is gratefully acknowledged. 


\section{References}

[1] Denton, J. D. The 1993 IGTI Scholar Lecture: Loss Mechanisms in Turbomachines. Journal of Turbomachinery, 1993, 115(4) pp 621-656.

[2] Paniagua, G., Iorio, M., Vinha, N., Sousa, J. Design and analysis of pioneering high supersonic axial turbines. International Journal of Mechanical Sciences, 2014, 89 pp 65-77.

[3] Lakshminarayana, B., Horlock, J. Review: Secondary flows and losses in cascades and axialflow turbomachines. International Journal of Mechanical Sciences, 1963, 5(3) pp 287-307.

[4] Langston, L. Secondary flows in axial turbines - a review. Annals of the New York Academy of Sciences, 2001, 934(1) pp 11-26.

[5] Sieverding, C. H. Recent Progress in the Understanding of Basic Aspects of Secondary Flows in Turbine Blade Passages. Journal of Engineering for Gas Turbines and Power, 1985, 107(2) pp 248-257.

[6] Wang, H. P., Olson, S. J., Goldstein, R. J., Eckert, E. R. G. Flow Visualization in a Linear Turbine Cascade of High Performance Turbine Blades. Journal of Turbomachinery, 1997, 119(1) pp 1-8.

[7] Dossena, V., Perdichizzi, A., Savini, M. The Influence of Endwall Contouring on the Performance of a Turbine Nozzle Guide Vane. Journal of Turbomachinery, 1999, 121(2) pp 200208.

[8] Moser, N., Steinhoff, P., Joos, F. Experimental and Numerical Investigations of Flowpath Profiling on Secondary Flow Losses in a Turbine Control Stage. In: ASME Turbo Expo 2013: Turbine Technical Conference and Exposition, American Society of Mechanical Engineers, 2013, ASME Paper GT2013-94737.

[9] Kumar, K. N., Govardhan, M. Secondary Flow Loss Reduction in a Turbine Cascade with a Linearly Varied Height Streamwise Endwall Fence. International Journal of Rotating Machinery, 2011, 2011(2011) pp 1-16.

[10] Rose, M. G. Non-axisymmetric endwall profiling in the HP NGV's of an axial flow gas turbine. In: ASME 1994 International Gas Turbine and Aeroengine Congress and Exposition, American Society of Mechanical Engineers, 1994, ASME Paper 94-GT-249.

[11] Hartland, J., Gregory-Smith, D., Harvey, N., Rose, M. Non-Axisymmetric Turbine End Wall Design: Part II-Experimental Validation. In: ASME 1999 International Gas Turbine and Aeroengine Congress and Exhibition, American Society of Mechanical Engineers, 1999, ASME Paper 99-GT-338.

[12] Ingram, G., Gregory-Smith, D., Rose, M., Harvey, N., Brennan, G. The effect of end-wall profiling on secondary flow and loss development in a turbine cascade. In: ASME Turbo Expo 2002: Power for Land, Sea, and Air, American Society of Mechanical Engineers, 2002, ASME Paper GT2002-30339.

[13] Brennan, G., Harvey, N., Rose, M., Fomison, N., Taylor, M. Improving the efficiency of the Trent 500-hp turbine using nonaxisymmetric end walls-Part I: Turbine design. Journal of turbomachinery, 2003, 125(3) pp 497-504.

[14] Harvey, N., Brennan, G., Newman, D., Rose, M. Improving turbine efficiency using nonaxisymmetric end walls: Validation in the multi-row environment and with low aspect ratio blading. In: ASME Turbo Expo 2002: Power for Land, Sea, and Air, American Society of Mechanical Engineers, 2002, ASME Paper GT2002-30337.

[15] Germain, T., Nagel, M., Raab, I., Schüpbach, P., Abhari, R. S., Rose, M. Improving Efficiency of a High Work Turbine Using Nonaxisymmetric Endwalls- Part I: Endwall Design and 
Performance. Journal of Turbomachinery, 2010, 132(2) pp 021007-021007.

[16] Schuepbach, P., Abhari, R., Rose, M., Germain, T., Raab, I., Gier, J. Improving Efficiency of a High Work Turbine Using Nonaxisymmetric Endwalls-Part II: Time-Resolved Flow Physics. Journal of Turbomachinery, 2010, 132(2) pp 021008.

[17] Harvey, N. W., Rose, M. G., Taylor, M. D., Shahpar, S., Hartland, J., Gregory-Smith, D. G. Nonaxisymmetric Turbine End Wall Design: Part I-Three-Dimensional Linear Design System. Journal of Turbomachinery, 2000, 122(2) pp 278-285.

[18] Praisner, T. J., Allen-Bradley, E., Grover, E. A., Knezevici, D. C., Sjolander, S. A. Application of Nonaxisymmetric Endwall Contouring to Conventional and High-Lift Turbine Airfoils. Journal of Turbomachinery, 2013, 135(6) pp 061006-061008.

[19] Da Lio, L., Manente, G., Lazzaretto, A. Predicting the optimum design of single stage axial expanders in ORC systems: Is there a single efficiency map for different working fluids? Applied Energy, 2016, 167 pp 44-58.

[20] Al Jubori, A. M., Al-Dadah, R., Mahmoud, S. Performance enhancement of a small-scale organic Rankine cycle radial-inflow turbine through multi-objective optimization algorithm. Energy, 2017, 131 pp 297-311.

[21] Meroni, A., Andreasen, J. G., Persico, G., Haglind, F. Optimization of organic Rankine cycle power systems considering multistage axial turbine design. Applied Energy, 2017.

[22] Song, Y., Sun, X., Huang, D. Preliminary Design and Performance Analysis of a Centrifugal Turbine for Organic Rankine Cycle (ORC) Applications. Energy, 2017.

[23] Al Jubori, A., Daabo, A., Al-Dadah, R. K., Mahmoud, S., Ennil, A. B. Development of microscale axial and radial turbines for low-temperature heat source driven organic Rankine cycle. Energy Conversion and Management, 2016, 130 pp 141-155.

[24] Sun, H., Li, J., Song, L., Feng, Z. Non-Axisymmetric Turbine Endwall Aerodynamic Optimization Design: Part I-Turbine Cascade Design and Experimental Validations. In: ASME Turbo Expo 2014: Turbine Technical Conference and Exposition, American Society of Mechanical Engineers, 2014, ASME Paper GT2014-25362.

[25] Tang, H., Liu, S., Luo, H. Design Optimization of Profiled Endwall in a High Work Turbine. In: ASME Turbo Expo 2014: Turbine Technical Conference and Exposition, American Society of Mechanical Engineers, 2014, ASME Paper GT2014-26190.

[26] Walraevens, R. E., Gallus, H. E. European Reasarch Community on Flow Turbulence and Combustion; ERCOFTAC SIG on 3D turbomachinary flow preditions; Test Case 6: 1-1/2 stage axial flow turbine. In: Seminar and Workshop on 3D Turbomachinary flow prediction, 1997.

[27] Taremi, F. Endwall Flows in Transonic Turbine Cascades. Carleton University, 2013, Ottawa. [28] Menter, F., Ferreira, J. C., Esch, T., Konno, B., Germany, A. The SST turbulence model with improved wall treatment for heat transfer predictions in gas turbines. In: Proceedings of the international gas turbine congress, 2003, 2-7.

[29] Wilcox, D. C. Companion Software: Turbulence Modeling for CFD, DCW Industries, Inc., 3rd Ed., 2006.

[30] Jasak, H., Beaudoin, M. OpenFOAM Turbo Tools: From General Purpose CFD to Turbomachinery Simulations. 2011, (44403) pp 1801-1812.

[31] Roache, P. J. Perspective: a method for uniform reporting of grid refinement studies. Journal of Fluids Engineering, 1994, 116(3) pp 405-413.

[32] Wilcox, D. C. Turbulence Modeling for CFD, 3rd Ed., DCW Industries, Inc., 2006.

[33] Piegl, L., Tiller, W. The NURBS book, Springer Science \& Business Media, 2012.

[34] Devore, J. L. Probability and Statistics for Engineering and the Sciences, Cengage Learning, 
2015.

[35] Kadhim, H. T., Rona, A., Obaida, H. M., Gostelow, J. P. Numerical Study of the Flow past an Axial Turbine Stator Casing and Perspectives for Its Management. In: ASME Turbo Expo 2017: Turbine Technical Conference and Exposition, 2017, ASME Paper GT2107-63055.

[36] Reutter, O., Hervé, S., Nicke, E. Automated optimization of the non-axisymmetric hub endwall of the rotor of an axial compressor. European Turbomachinery Conference, Lapeenranta, 2013.

[37] Persico, G. Evolutionary Optimization of Centrifugal Nozzles for Organic Vapours. In: Journal of Physics: Conference Series, IOP Publishing, 2017, 012015.

[38] Alstom, The ALSTOM Process \& Optimisation Workbench, User Manual, GE Power, (version 4.0), 2014.

[39] Gallus, H. E., Zeschky, J. Secondary Flow and Losses in a Turbine Rotor at Off-Design Conditions In: Cieplne Maszyny Przepływowe (CMP), 1992. 